\title{
Paradis d'Abdulrazak Gurnah : une culture de l'échange
}

\section{Paradis by Abdulrazak Gurnah: a Culture of Exchange}

Eve LÉGER-BÉLANGER [eve.leger-belanger@mail.mcgill.ca]

Université de Montréal, Canada

\section{RÉSUMÉ}

Notre article se propose d'étudier les manières dont la colonisation bouleverse le monde de l'échange omniprésent dans le roman Paradis d'Abdulrazak Gurnah. Nous analysons la culture de l'échange tant en ce qui a trait au fond qu'à la forme du roman. Nous examinons le rôle des individus, mais aussi des lieux, comme la ville, dans le processus de contact entre les personnages. Nous explorons également le jeu dans la forme même du récit, qui devient un échange entre divers genres littéraires. Nous fondons notre approche théorique sur les ouvrages de Pierre Halen qui s'attache à l'identité et la colonisation, de Dominique Maingueneau qui étudie le discours et le genre, et de Jean-Marie Schaeffer qui interroge les relations entre le texte et le genre.

\section{MotS-CLÉS}

roman ; transculture ; échange ; forme ; genre ; littérature francophone

\begin{abstract}
Our paper investigates how colonization disrupts the world of exchange in the novel Paradise by Abdulrazak Gurnah. We analyze the culture of exchange both in regard to the content and the formal construction of the novel. We examine the role of the individuals and the places like the city in the contact process between people. We also explore how the narrative becomes an exchange between various literary genres. We base our theoretical approach on the works of Pierre Halen linking identity and colonization, Dominique Maingueneau specialized in discourse analysis and genre, and Jean-Marie Schaeffer who questions the relationship between the concept of text and genre.
\end{abstract}

\section{KEYWORDS}

novel; transculture; exchange; novel form; genre; Francophone literature

REÇU 2015-08-31 ; ACCEPTE 2016-06-30 
Il découle de la colonisation une mise en contact entre des cultures, le plus souvent très différentes, qui se trouvent à être confrontées à des systèmes de valeurs discordants. De même, la colonisation conduit à une ère de changement profond pour un pays. Cette présence imposée par le colonisateur - pacifique ou non - crée une remise en question de l'identité du colonisé. Dans ce contexte, les individus en viennent à se définir par la différenciation avec les autres.

L'auteur tanzanien Abdulrazak Gurnah ${ }^{1}$ met en place cet univers où des habitants africains se heurtent à la colonisation européenne. Dans son œuvre et plus particulièrement dans le roman Paradis $^{2}$, Gurnah développe le thème de la tension entre la perception individuelle de la colonisation et ce qui en a été retenu par la collectivité. Dans Paradis, les habitants de la Tanzanie et du Zanzibar se heurtent à la colonisation européenne, plus particulièrement celle des Allemands et des Anglais, bien que celle des Belges soit aussi présente. La perception individuelle du colonisé à propos de cette ère de changement causée par la colonisation est, dans le cas de Paradis, celle de Yusuf, un jeune tanzanien qui grandit au cœur de l'expansion coloniale en Tanzanie et de l'ajustement forcé des valeurs par les Africains.

En quoi le roman Paradis est-il l'incarnation d'une culture de l'échange pluriel ? De quelles façons la transculture ${ }^{3}$ prend-elle forme et se manifeste-t-elle sur le plan textuel ? Nous considérons que cet échange se traduit tant en ce qui a trait au fond qu'à la forme dans le roman.

Dans un premier temps, nous analyserons les manières dont les personnages de différentes ethnies cohabitent, par l'écoute et la différenciation entre les peuples. Nous déclinerons le motif de la migration par la cohabitation, le commerce et le mélange des langues. Nous grefferons à cette culture de l'échange la présence des Européens et leur rôle dans le processus de colonisation. Dans un deuxième temps, nous étudierons la notion d'échange dans le roman à travers la forme même du texte et à travers le jeu avec le genre.

Nous fondons notre approche sur divers modèles théoriques. Nous avons été influencés par des ouvrages qui portent sur la théorie du langage, puisque l'objet de notre étude comprend une analyse du discours et une observation des concepts de langue et de traduction. Nous nous sommes essentiellement fondés sur la théorie de Dominique Maingueneau et plus particulièrement sur l'ouvrage Le discours littéraire, afin d'analyser les actes d'énonciation et le jeu avec la langue dans le roman. Ces actes se trouvent dans un univers discursif qui est « l'ensemble des formations discursives de tous type qui interagissent dans une conjoncture donnée » (Maingueneau 2004 : 27). Nous nous concentrerons ainsi sur les objets qui interagissent au sein d'un lieu particulier, dans notre cas, le roman. L'article «Construction identitaires et stratégies d'émergence: notes pour une analyse institutionnelle du système littéraire francophone » de Pierre Halen nous sera également utile en ce qui a trait à sa réflexion sur le métissage des cultures. Nous nous appuierons aussi sur Qu'est-ce qu'un genre littéraire par Jean-Marie Shaeffer pour remettre en question la relation entre le texte et son genre.

1 Professeur à l'université de Kent en Angleterre, Gurnah est l'auteur de huit romans dont le dernier, The Last Gift, est paru en 2011. Dans ses écrits, Gurnah s'intéresse surtout à la période du post-colonialisme.

2 Le roman a été publié initialement en anglais en 1994 et a été traduit en français en 1995.

3 Nous entendons par transculture un échange entre des cultures, une intégration de certaines valeurs par au moins l'une d'entre-elles et le refaçonnement de certains traits. 


\section{Les colonisés : la cohabitation en ville}

Les personnages dans Paradis sont de nationalités diverses, surtout en milieu urbain. Dans le récit, la migration est déjà un fait accompli. La narration révèle ainsi les résultats de cette migration, par des personnages qui habitent déjà au cœur de la même ville et qui doivent cohabiter. Se côtoient en milieu urbain des Africains natifs - dits civilisés ou sauvages -, des Grecs, des Indiens et des Arabes. La ville est donc un espace « local » ou le multiculturel international est chose habituelle.

De fait, plusieurs Africains natifs et vivant en milieu urbain se jugent civilisés par rapport à leurs pairs qui vivent dans les profondeurs du pays, " au pays des sauvages" » (Gurnah 1995 : 38). Le système hiérarchique entre les natifs est donc clairement défini. Le protagoniste, Yusuf, provient de ces "terres sauvages » (77) et se le fait sans cesse rappeler : " Mais tu sais déjà tout ça, tu en viens de ce pays de sauvages! » (77). Cependant, Yusuf a quitté ces contrées dites sauvages pour se joindre à la vie de commerce d'un personnage nommé l'oncle Aziz. En vivant désormais en ville et en participant aux voyages des caravanes pour faire du commerce, le protagoniste atteint le statut de « civilisé ».

Les Indiens constituent par ailleurs un groupe ethnique prépondérant dans le récit. Cette communauté interagit le plus souvent avec celle des Africains par la différenciation, qui plus est, par la différenciation négative. Des enfants indiens insultent par exemple Yusuf (20) en raison de sa nationalité différente de la leur. Les Indiens sont pensés par les autres personnages comme des individus non dignes de confiance : «Il ne faut jamais se fier à un Indien » (177). Le contact avec l'autre passe donc dans le cas des relations avec la communauté indienne par une fonction repoussoir entre les nationalités.

\section{Les colonisateurs : la présence distante mais marquante}

En plus des Africains - dits civilisés ou sauvages -, des Indiens, des Arabes, etc. qui habitent en Tanzanie, un nouveau groupe vient s'établir sur le territoire déjà multiculturel : celui des Européens. Dès les premières pages du roman, la présence des Européens en Tanzanie est présentée de façon figurative : "lénorme drapeau jaune portant l'emblème d'un éclatant oiseau noir » (14). Les colonisateurs sont ici initialement incarnés par le biais d'un symbole de souveraineté en un lieu donné, le drapeau, et non en tant qu'individus permettant l'échange, ce qui est le cas entre les Africains natifs, les Arabes, etc. Il ne s'agit donc pas cette fois de cohabitation, mais plutôt d'une relation entre le dominant, le colonisateur, imposant son point de vue au dominé, le colonisé.

Il résulte de la domination européenne un décalage dans le système de lois. Par exemple, un épisode dans le récit révèle la façon dont un Africain ne connaît pas les lois du nouveau gouvernement colonisateur au pouvoir (214) et ne comprend pas ce nouveau système imposé, ce qui crée un quiproquo dans ses démarches administratives. En ce sens, les colonisés n’ont pas encore intégrés de façon fonctionnelle le système administratif occidental : il s'agit encore d'une période de transition. Il semble donc y avoir une imposition du système de valeurs, de

4 Les renvois au roman se feront désormais entre parenthèse uniquement par la mention à la page citée. 
fonctionnement et de lois des Européens, plus particulièrement des Allemands, sur les Tanzaniens. Il n'y a pas d'échange dans cette relation; il s'agit plutôt d'un transfert unidirectionnel.

Malgré la présence de signes directement perceptibles des colonisateurs en sol africain, tels les drapeaux, le système européen est présenté dans la narration comme étant régit par une entité lointaine. En effet, même s'ils doivent obéir au nouvel ordre social imposé par les colonisateurs, la plupart des Tanzaniens n'ont jamais été en interaction directe avec des Européens. Il en découle chez les colonisés de nombreux récits à propos des êtres puissants que représentent les colonisateurs :

Dans le pays mystérieux des guerriers, de l'autre côté de la montagne, où la pluie était rare, vivait un Européen légendaire. On le disait très riche. Il avait appris le langage des animaux, pouvait parler avec eux et leur donner des ordres. Son royaume sétendait sur de vastes étendues, et son palais de fer, construit sur une falaise, était aussi un puissant aimant (90).

Ces histoires collectives font des Allemands des êtres quasi-mythiques : «Était-ce vrai qu'ils pouvaient avaler du métal? »(213). Les histoires racontées sur les Européens les dépeignent comme des êtres sans merci, féroces et brutaux, dont la salive serait empoisonnée (101). L'imaginaire des colonisés renvoie donc à une puissance extrême, mais négative.

Au niveau concret, les colonisés sont conscients du changement profond dans leur mode de vie que la colonisation par les Européens suscitera. D’un côté, les Tanzaniens croient que les occidentaux imposeront leur mode de vie complet aux jeunes et déformeront la culture actuelle de la Tanzanie :

Nous allons tout perdre et aussi notre manière de vivre. Les jeunes seront les grands perdants : il viendra un jour où les Européens les feront cracher sur tout ce que nous savons, et les obligeront à réciter leurs lois et leur histoire du monde comme si c'était la parole sacrée. Quand, un jour, ils écriront sur nous, que diront-ils? Que nous avions des esclaves (120).

D’un autre côté, certains Tanzaniens pensent que le changement créé par la colonisation n'est pas nécessairement quelque chose de négatif et que la vie continuera son cours :

Pourquoi être si pessimistes, protesta Hamid. Est-ce que notre manière de vivre est si merveilleuse que ça? Nous avons déjà trop de soucis pour se préoccuper de ces sombres prédictions. Laissons tout ça dans les mains de Dieu. Les choses changeront peut-être, mais le soleil se lèvera toujours à l'est et se couchera à l'ouest. Parlons d'autre chose (120).

En ce sens, il ne faudrait pas se préoccuper outre mesure d'un changement impossible à empêcher. La colonisation est donc une occupation imposée qui est cependant comprise par les colonisés dans sa complexité positive et négative. Ainsi, qu'il soit de direct ou indirect, le contact entre les individus est omniprésent dans le récit. Halen résume ce contact en mentionnant que « la culture (ce qui se cultive historiquement) est toujours interculturelle; elle n'est pas l'Un qui devrait, au nom d'une morale ou d'une politique, se mettre à dialoguer avec un Autre : elle est, dans son essence, déjà dialogue » (Halen 2001 : 17). Reste que, ce dialogue peut être inégal. 


\section{L'échange par les biens : le commerce}

Par l'échange de marchandises, le commerce sous-tend aussi un échange entre des individus. Dans le cas de Paradis, cet échange se fait entre des personnages de pays distincts, ce qui crée une confrontation entre des cultures différentes. À ce titre, plusieurs marchands grecs et indiens tiennent des commerces en ville (97). Les Indiens sont les commerçants les plus puissants en ville, plus puissants que les Africains (220), ce qui explique leur dépréciation dans le récit, en raison de la forte compétition créée par le commerce.

Une caravane dirigée par l'Oncle Aziz, qui va dans les profondeurs de l'Afrique pour «faire du commerce avec les sauvages » (53), est une autre voie dans le récit qui illustre le lien inextricable entre l'échange de biens et la culture de l'échange. D'ailleurs, la distinction entre la civilisation et l'état sauvage est, selon les " civilisés », la capacité par les individus à faire du commerce (77). Les personnes sans commerce sont de fait associées par les commerçants à des « insectes paralysés » (159). Le commerce, synonyme de civilisation, crée donc un milieu interculturel par l'échange qu'il suscite.

Les colonisateurs ne sont pas en reste pour réclamer leur part du commerce en Tanzanie. Ils modernisent le négoce en misant sur la construction d'un chemin de fer qui permettra une efficacité plus rentable que les caravanes. Hormis une mention à des fermiers européens (97), les Européens sont les employeurs lointains et les personnages non européens constituent la main d'œuvre. D’ailleurs, celui qui maîtrise le transport maîtrise le commerce : «Et maintenant on parle de ces Allemands, qui construisent une voie ferrée jusqu'ici. C’est eux qui font la loi désormais » (175). En prenant en charge le commerce, les colonisateurs créent un changement dans les racines mêmes de l'identité des colonisés, puisque le commerce est central dans leur vie et vital dans leur définition de civilisation. Les colonisateurs bousculent donc le fonctionnement des relations entre les autres personnages ; ils changent le fonctionnement de la culture de l'échange qui était établie. Les natifs sont conscients que, sous peu, ils n’auront plus accès à leur propre territoire pour le commerce, en raison du monopole établi par les colonisateurs européens : « Nous retournerons à Marungu l'an prochain, avant que la région ne soit envahie par les Belges, qui vont bientôt nous interdire d'y aller» (124). Malgré les emplois que la colonisation suscite, le discours des colonisés est parsemé de commentaires négatifs à l'égard des Européens qui prennent en charge le commerce : « ils nous écraseront tous » (118); « ils convoitent le monde entier » (119). Les verbes d'action « écraser » et « convoiter » mettent l'accent sur le dynamisme négatif des colonisateurs en conquête de territoires. Cependant, aucun indice dans le récit ne laisse croire que les colonisés résistent à l'instance qui les colonise; ils ne font que constater et juger.

\section{L'échange par la communication : la langue}

Outre le commerce, la langue est un vecteur d'échange central dans le roman. Par exemple, l'Oncle Aziz et Khalil, l'ami arabe de Yusuf, se parlent en arabe. Or, Yusuf ne comprend pas cette langue (37) et se trouve donc exclu de la discussion. Ces épisodes ponctuels dans le récit mettent en relief le fait que l'apprentissage des langues est pensé par les personnages comme 
un atout pour l'individu : «Il faut que tu apprennes l'arabe, ça te ferait bien voir de lui [l'Oncle Aziz]» (43). La compréhension de plusieurs langues n'est pas l'unique atout mis en relief, le son même de celles-ci néchappe pas aux personnages. Par exemple, l'attention des personnages est aussi fixée sur la prononciation : " Il [Khalil] parlait couramment le kiswahili, mais avec un fort accent arabe » (39). L'attention portée à l'accent des personnages contribue à nourrir la dimension d'oralité liée à la langue parlée : « La littérature orale ne délivre donc un sens que porté par un rythme; la voix y possède une épaisseur, elle atteint tous les registres sensoriels des auditeurs pour susciter la communion. On est fort loin de la littérature imprimée » (Maingueneau 2004 : 166). La description du son donne en ce sens un caractère oral au récit ici mis sur papier. Les langues ne sont du reste pas pensées comme purement utilitaire. En effet, même si la compréhension de plusieurs langues est un atout, les personnages sont ouverts à la mise en contact avec différentes langues sans que la compréhension de celles-ci ne soit nécessaire : «Personne dans la foule ne comprenait les paroles des mélodies, mais tous restaient pour les écouter » (76), «Ils n’étaient pas vraiment silencieux, mais parlaient entre eux une langue qu'ils étaient seuls à comprendre » (52). Ce jeu de contact entre les langues des personnages sert de rappel implicite à la transculture qui se profile dans le récit en raison des migrations faites par le passé et du contact entre les diverses communautés qui en résulte.

En plus des mentions à la diversité des langues, la notion de traduction est omniprésente dans le texte. De fait, les dialogues dans le récit impliquent souvent des traductions simultanées. La traduction en français est le plus souvent accolée à l'expression dans une autre langue : "Kijana mzuri », " joli garçon », dit Mohammed en s'arrêtant prêt de Yusuf » (77) ; "Après le kiyama, le jour de la fin du monde où Dieu appelle tous les hommes à lui » (47). Le rythme des prises de parole par les personnages et le rythme de la narration sont ralentis par cette traduction. Cependant, grâce à ce mode d'expression dans les discussions, les personnages se familiarisent à une culture étrangère, par l'entremise de la langue, que ce soit par la traduction ou par le contact avec une sonorité différente.

S'ajoutent à la narration des termes qui ne sont pas en français et qui ne sont pas traduits. Les termes qui ne sont pas en français se trouvent le plus souvent dans les énumérations : «Il emmenait souvent avec lui des joueurs de tambour, de tamburi, de cor et de siwa; quand le cortège entrait dans la ville, les animaux affolés s'enfuyaient et les enfants venaient à sa rencontre » (16). Le lecteur francophone peut comprendre le sens des termes employés, même s'ils ne sont pas traduits, grâce à l'effet d'accumulation créé par les mots en français qui entourent les termes étrangers.

Dans un texte, l'auteur est en maîtrise de la langue, ou des langues, au(x)quelle(s) il a recourt : "L'écrivain n'est pas confronté à la langue, mais à une interaction de langues et d'usages, à ce qu'on appellera une interlangue [...] En fonction de l'état du champ littéraire et de la position qu'il occupe, l'écrivain négocie à travers l'interlangue un code langagier qui lui est propre » (Maingueneau 2004 : 140). Le lecteur a donc accès à l' " interlangue » spécifique de Gurnah qui joue avec les divers niveaux d'interaction d'une langue, des langues, ce qui nourrit le motif de la culture de l'échange non seulement en ce qui a trait au thème, mais aussi en ce qui concerne la manière d'exprimer ce thème par les mots en soi. 


\section{Léchange dans la forme : le jeu avec le genre}

Sur la quatrième de couverture du livre, Paradis est présenté comme un roman, ce qui le classe dans un genre littéraire donné avant même le début de la lecture. En un certain sens, cette classification fige le texte dans un carcan donné : «En attribuant telle étiquette à telle œuvre, on indique comment on prétend que son texte soit reçu, on instaure une manière non négociée un cadre pour une activité discursive » (Maingueneau 2004 : 181). Il est malgré tout possible pour l'auteur de manier les frontières du genre même si celui-ci est initialement défini : "Létiquette ainsi conférée par l'auteur ne caractérise qu'une part de la réalité communicative du texte [...] car le roman [...] participe d'une littérature qui est affectée d'une indétermination générique constitutive » (Maingueneau 2004 : 184). Dans la même veine, Schaeffer réfléchit au phénomène englobant du genre qui comprend des variations internes :

Quel que soit le cheminement choisi, le phénomène est toujours le même : les termes génériques se référant à des traits textuels sont passibles d'une logique de la différenciation interne, en ce sens que le texte individuel n'exemplifie pas simplement des propriétés fixées par le nom de genre, mais module sa compréhension, c'est-à-dire institue et modifie les propriétés pertinentes. On passe de l'identification générique exemplifiante à l'identification générique modulatrice (Shaeffer 1989 : 166).

Ainsi, malgré son appartenance initiale au genre romanesque, Paradis est doté de variations internes du genre qui lui donnent une structure complexe en ce qui a trait à la forme. Reste à déterminer quelles sont ces variations.

Outre l'appartenance au genre romanesque, Paradis peut être pensé comme une série de nouvelles. En effet, le roman est divisé en sections distinctes, séparées par une page vierge recto verso avec le nom de la section imprimé au recto. Ces sections visuellement distinguables peuvent théoriquement fonctionner de façon indépendante, même si le regroupement en un seul roman permet une continuité entre celles-ci et donne une logique évolutive au récit.

Paradis s'apparente également à un récit de formation. En effet, le récit principal est celui d'un jeune adolescent, Yusuf, qui est en constante observation du monde qui l'entoure, ce qui lui permet d'évoluer par le biais du contact avec autrui. Il est d'ailleurs directement question dans la narration de récit de formation : «il [Yusuf] pourrait même leur rendre visite, et les [Hamid et sa femme] remercier de l'avoir mis à rude école pour le préparer à la vie» (287). De plus, le passage du temps dans le récit est indiqué par des mentions à l'âge de Yusuf. Lâge du personnage qui devient adulte et formé devient dès lors le barème temporel. Le jeune Yusuf se fait éduquer par d'autres personnages plus vieux et plus expérimentés que lui, comme par un certain Mohammed : «Tu [Yusuf] vas faire du commerce, apprendre la différence entre une vie civilisée et la vie que mènent les sauvages. Il est temps que tu grandisses et que tu vois à quoi ressemble le monde » (77). La formation du jeune homme se fait donc par l'échange avec autrui. Les apprentissages de Yusuf passent d'ailleurs autant par le commerce que par la maîtrise de la lecture et de la mécanique (139-140) : tant le côté intellectuel que pratique sont couverts. Cet apprentissage ne se fait toutefois jamais seul. Au même titre que le récit de formation, le roman 
fait penser à une biographie, quoiqu'ici fictive, puisque l'on raconte essentiellement la vie d'un seul personnage : Yusuf.

Paradis se rattache aussi, de façon secondaire, au conte. En effet, malgré les quelques rares indices temporels tels que l'âge du protagoniste et les indices implicites qui renvoient à la période de la colonisation par les Européens en Tanzanie, la narration omet presque toute référence temporelle directe, ce qui rappelle l'imaginaire du conte se trouvant habituellement dans une sorte de hors temps. Certains éléments magiques introduits dans le récit renforcent cette idée du conte, comme la certitude de la maîtresse d'Oncle Aziz qui croit que Yusuf possède un don où tout ce qu'il touche fleurit et guéri (258). Cette magie déplace le récit de la "réalité fictive » au conte où le merveilleux est possible. De même, les nombreuses histoires orales intégrées au récit, comme les pouvoirs "maléfiques » des Européens, nourrissent l'univers du conte établi dans le texte.

Le jeu entre la fiction et le réel prend également forme dans Paradis par une intertextualité. Les notes en bas de page servent par exemple à donner des détails sur un personnage historique mentionné dans la narration, tel "Iskander le Conquérant " invoqué en bas de page comme "Alexandre Le Grand »(62). Les notes en bas de pages servent essentiellement à clarifier des références implicites, par exemple lorsque Yusuf se fait déchirer la chemise par la maîtresse de l'Oncle Aziz : « il [Khalil] jeta un coup d'oil sur le dos de Yusuf, ne put retenir un rire nerveux et resta un moment pensif; cela lui rappelait quelque chose ». Cette phrase est suivie d'un astérisque développant l'association sous-entendue : "Allusion au Yusuf de la XIIe sourate du Coran : sa chemise a été déchirée par-derrière par Zulekha, l'épouse de l'intendant du pharaon. Cf. Genèse, ch. 39, où Joseph se trouve dans la même situation » (291). Les explications en bas de pages sont ainsi dédiées au lecteur qui est appelé à créer des liens entre le récit même et des éléments externes à celui-ci : « le texte n'est pas destiné à être contemplé, il est énonciation tendue vers un coénonciateur qu'il faut mobiliser pour le faire adhérer "physiquement » à un certain univers de sens " (Maingueneau 2004 : 201), ce qui rend l'échange entre le texte et le lecteur actif. Par conséquent, l'intertextualité crée un dialogue, une réciprocité, entre le texte, ses renvois et le lecteur.

En somme, Paradis est un roman qui transcende de façon interne les limites du genre. Le genre romanesque est, selon l'appellation de Schaeffer, l' " acte communicationnel enveloppant » (Shaeffer 1989 : 162). Cet " acte communicationnel peut englober d'autres actes communicationnels ou être englobé par eux, sans que cette hiérarchie entame la logique de l'exemplification générique globale propre à chacun » (Shaeffer 1989 : 162). En ce sens, Paradis serait à la fois un récit de formation, un conte, des nouvelles, et ce, dans une perspective d'échange dynamique au sein du genre romanesque englobant. Il y a donc un jeu avec les formes (récit de formation, biographie, conte, etc.) et un métissage nouveau au sein d'une forme précise, le roman. Ce processus de l'échange dans la forme même du roman soulève la traditionnelle séparation contenant-contenu, qui cette fois se complètent. Or on dépasse de loin ici la binarité, pour rejoindre plutôt une toile d'araignées de référents tant thématiques que formels, et c'est là que l'imbrication de tout cela devient intéressante. 
Si on se questionne sur la publication de Paradis dans le monde contemporain, on comprend que cette œuvre n'a pas une valeur purement fictive. Il s'agit aussi d'un récit social et politique. La confrontation avec l'autre qui définit l'identité du colonisé est un thème actuel pour la Tanzanie, dont l'indépendance est relativement récente (1964). La colonie a été créée en 1880 (protectorat allemand) et a été conquise par les armées britanniques et belges après la Première Guerre mondiale. La Tanzanie a connu pendant de nombreuses années ce protectorat imposé, devenant un prix de victoire sans consentement de la part des colonisés. Ces derniers ont donc dû choisir entre accepter, rejeter ou s'adapter à l'influence extérieure. Malgré la déclaration d'indépendance en 1964, la confrontation aux valeurs occidentales est encore d'actualité pour les Tanzaniens qui se trouvent à être constamment en contact avec la culture occidentale qui tend à être ethnocentriste et à vouloir dominer l'ailleurs. Paradis est donc aussi une mise sur papier imaginaire du malaise identitaire constant et réel pour les Tanzaniens, exacerbé par certaines conditions historiques.

\section{Références bibliographiques}

\section{Cuvre à l'étude}

Gurnah, A. (1995). Paradis. Trad. A. C. Padoux. Paris : Denoël.

\section{Ouvrages théoriques}

Halen, P. (2001). Constructions identitaires et stratégies d'émergence : notes pour une analyse institutionnelle du système littéraire francophone. Études françaises, 37, 13-31.

Maingueneau, D. (2004). Le discours littéraire : paratopie et scène d'énonciation. Paris : A. Colin.

Shaeffer, J.-M. (1989). Qu'est-ce qu’un genre littéraire. Paris : Seuil. 
